\title{
Alteration of matrix metalloproteinase-3 O-glycan structure as a biomarker for disease activity of rheumatoid arthritis
}

Masaru Takeshita ${ }^{1 \dagger}$, Atsushi Kuno ${ }^{2,3 \dagger}$, Katsuya Suzuki ${ }^{1}$, Atsushi Matsuda ${ }^{2,3}$, Hiroko Shimazaki ${ }^{2}$, Tomomi Nakagawa ${ }^{4}$, Yuki Otomo ${ }^{1}$, Yasuaki Kabe ${ }^{4}$, Makoto Suematsu ${ }^{4}$, Hisashi Narimatsu ${ }^{2}$ and Tsutomu Takeuchi ${ }^{1 *}$

\begin{abstract}
Background: Nearly all secreted proteins are glycosylated, and serum glycoproteins that exhibit disease-associated glycosylation changes have potential to be biomarkers. In rheumatoid arthritis (RA), C-reactive protein (CRP), and matrix metalloproteinase-3 (MMP-3) are widely used as serologic biomarkers, but they lack sufficient specificity or precision. We performed comparative glycosylation profiling of MMP-3 using a recently developed antibody-overlay lectin microarray technology that allows semicomprehensive and quantitative analysis of specific protein glycosylation to develop an RA-specific disease activity biomarker.

Methods: Serum was taken from patients with RA ( $n=24)$ whose disease activity was scored using composite measures, and MMP-3 was immunoprecipitated and subjected to lectin microarray analysis. A disease activity index (DAI) based on lectin signal was developed and validated using another cohort $(n=60)$. Synovial fluid MMP-3 in patients with RA and patients with osteoarthritis (OA) was also analyzed.

Results: Intense signals were observed on a sialic acid-binding lectin (Agrocybe cylindracea galectin [ACG]) and O-glycan-binding lectins (Jacalin, Agaricus bisporus agglutinin [ABA], and Amaranthus caudatus agglutinin [ACA]) by applying subnanogram levels of serum MMP-3. ACG, ABA, and ACA revealed differences in MMP-3 quantity, and Jacalin revealed differences in MMP-3 quality. The resultant index, ACG/Jacalin, correlated well with disease activity. Further validation using another cohort confirmed that this index correlated well with several DAls and their components, and reflected DAl changes following RA treatment, with correlations greater than those for MMP-3 and CRP. Furthermore, MMP-3, which generated a high ACG/Jacalin score, accumulated in synovial fluid of patients with RA but not in that of patients with OA. Sialidase digestion revealed that the difference in quality was derived from O-glycan a-2,6-sialylation. Conclusions: This is the first report of a glycoprotein biomarker using glycan change at a local lesion to assess disease activity in autoimmune diseases. Differences in the degree of serum MMP-3 a-2,6-sialylation may be a useful index for estimating disease activity.
\end{abstract}

Keywords: Rheumatoid arthritis, Glycoprotein, Biomarker, MMP-3

\footnotetext{
* Correspondence: tsutake@z5.keio.jp

${ }^{\dagger}$ Equal contributors

${ }^{1}$ Division of Rheumatology, Department of Internal Medicine, Keio University

School of Medicine, 35 Shinanomachi, Shinjuku-ku, Tokyo 160-8582, Japan

Full list of author information is available at the end of the article
} 


\section{Background}

Rheumatoid arthritis (RA) is a common autoimmune disease characterized by synovial inflammation and hyperplasia. The hyperplastic synovium produces vast amounts of matrix metalloproteinases (MMPs) that degrade cartilage matrix as well as synovial inflammatory cytokines such as macrophage colony-stimulating factor and receptor activator of nuclear factor $\mathrm{kB}$ ligand that promote osteoclastogenesis and disrupt joint structure [1].

To prevent RA progression, it is important to define the treatment target, such as remission or at least low disease activity; to assess disease activity using composite measures; and to adapt therapy if the target is not achieved within a particular time frame. This is referred to as a treat-to-target strategy [2, 3]. The Disease Activity Score in 28 joints (DAS28), which combines evaluation by a rheumatologist, laboratory test results, and the patient global assessment, has commonly been used to assess disease activity $[4,5]$. Recently, new indices such as the Simplified Disease Activity Index [6] and Clinical Disease Activity Index [7], which simplified the DAS28, have been developed.

C-reactive protein (CRP) and MMP-3 are widely measured as serum markers. Although CRP, an acute phase protein, reacts to joint inflammation, it cannot distinguish RA activity and other inflammatory conditions such as infectious disease. In contrast, MMP-3 is characterized as a more specific indicator of synovial inflammation. It was originally identified as a protein secreted from RA synovial fibroblasts [8]. MMP-3 degrades various extracellular substrates, including proteoglycan, fibronectin, laminin, and type 4 collagen, in addition to activating pro-MMPs. Thus, MMP-3 is thought to contribute to cartilage destruction in RA pathophysiology [9]. Serum MMP-3 is elevated in diseases that involve joint synovitis, including RA, reactive arthritis, psoriatic arthritis, and crystal arthritis, but not in osteoarthritis (OA) or systemic inflammatory conditions such as sepsis $[10,11]$. However, correlation with disease activity indices (DAIs) is superior in acute phase proteins compared with serum MMP-3 [12, 13]. Thus, development of an RA-specific disease activity biomarker is needed.

It is known that almost all secreted proteins are glycosylated, that glycosylation patterns are influenced by cellular differentiation, and that serum glycoproteins exhibiting disease-associated glycosylation changes have potential to be biomarkers [14]. For example, serum $\alpha$-fetoprotein (AFP), a commonly used hepatocellular carcinoma biomarker, can be fractionated into three glycosylation patterns-L1, L2, and L3-using Lens culinaris agglutinin lectin. Because AFP-L3 is produced only by hepatocellular carcinoma, measurement of AFP-L3 rather than total AFP provides superior sensitivity and specificity $[15,16]$.
Although analysis of carbohydrate chains has been difficult because of their repetitive sequence and structural variety, the recently developed antibody-overlay lectin microarray technology allows semicomprehensive and quantitative analysis of protein glycosylation patterns [14]. Kuno et al. [17] showed that the glycosylation pattern of serum Mac-2-binding protein, which had previously been reported as a quantitative marker for tumor progression and metastasis [18], gradually changes during liver fibrosis progression and thus serves as a biomarker for liver fibrosis.

In the present study, we focused on an existing biomarker, MMP-3, and examined the association between its glycosylation pattern and RA disease activity. We report on a new, sensitive biomarker that is based on local inflammation and can be assessed using protein glycosylation changes.

\section{Methods}

\section{Patients and samples}

RA serum and synovial fluid samples were collected at Keio University Hospital. All patients fulfilled the 2010 American College of Rheumatology/European League Against Rheumatism classification criteria for RA [19]. Written informed consent was obtained from all individuals. This study was approved by the institutional review board of Keio University School of Medicine and the National Institute of Advanced Industrial Science and Technology, and it was conducted in compliance with the Declaration of Helsinki.

\section{Sample preparation}

Serum MMP-3 enrichment was performed as previously described [14]. Serum or synovial fluid samples were precleared with 100- $\mu$ g streptavidin beads (FG beads; Tamagawa Seiki, Iida, Japan) at $4{ }^{\circ} \mathrm{C}$ for 30 minutes in Tris-buffered saline containing $1 \%$ Triton X-100 (TBS-Tx). Protein G-purified biotinylated anti-MMP-3 antibody (200 ng; R\&D Systems, Minneapolis, MN, USA) was added to precleared samples and incubated overnight at $4{ }^{\circ} \mathrm{C}$. Antibody complexes were incubated with $100-\mu \mathrm{g}$ streptavidin beads at $4{ }^{\circ} \mathrm{C}$ for 30 minutes, washed three times with TBS-Tx. MMP-3 and biotinylated anti-MMP-3 antibody were eluted by boiling at $95{ }^{\circ} \mathrm{C}$ for 5 minutes in Tris-buffered saline containing $0.2 \%$ sodium dodecyl sulfate. Antibodies were removed by incubation with $100-\mu \mathrm{g}$ streptavidin beads at $4{ }^{\circ} \mathrm{C}$ for 30 minutes. Supernatants were collected and used for the next assay as immunoprecipitation (IP) samples. For sialic acid digestion, IP samples were incubated with Glyko Sialidase A (PROzyme, Hayward, CA, USA) at $37{ }^{\circ} \mathrm{C}$ for $1 \mathrm{~h}$ before applying them to lectin microarrays (LecChip; GlycoTechnica, Yokohama, Japan). 


\section{Western blot analysis}

IP samples and a recombinant MMP-3 protein standard (Abcam, Cambridge, UK) were isolated by 10-20 \% SDSPAGE and transferred onto polyvinylidene difluoride membranes. Membranes were blocked with Block Ace (DS Pharma Biomedical, Osaka, Japan) for $1 \mathrm{~h}$, followed by incubation with biotinylated anti-MMP-3 antibody and streptavidin-HRP conjugate (Jackson ImmunoResearch, West Grove, PA, USA). After extensive washing, MMP-3 bands were semiquantified using ImmunoStar LD (Wako, Osaka, Japan).

\section{Antibody-overlay lectin microarray}

Antibody-overlay lectin microarray assays were performed as described previously [20,21]. Briefly, IP samples were diluted to $60 \mu \mathrm{l}$ with PBS containing $1 \%$ Triton X-100 (PBS-Tx) and applied to lectin microarrays. After incubation overnight at $20^{\circ} \mathrm{C}$, human serum immunoglobulin G (IgG) $(2 \mu \mathrm{l}$, approximately $10 \mathrm{mg} / \mathrm{ml}$; Sigma-Aldrich, St. Louis, MO, USA) was added to the array, followed by 30 minutes of incubation. The reaction solution was discarded, and the array was washed with PBS-Tx. Biotinylated anti-MMP-3 antibody (100 ng) was applied to the array, incubated at $20{ }^{\circ} \mathrm{C}$ for $1 \mathrm{~h}$, washed with PBS-Tx, and incubated at $20{ }^{\circ} \mathrm{C}$ for 30 minutes with $200 \mathrm{ng}$ of cyanine 3-labeled streptavidin (GE Healthcare, Little Chalfont, UK). The array was rinsed with PBS-Tx and scanned using an evanescent-field fluorescence scanner (GlycoStation; GlycoTechnica). All data were analyzed with ToolsPro Suite software V1.5 (GlycoTechnica). Net intensity was calculated by subtracting the mean background from the mean signal intensity of three spots per lectin. A schematic overview of this assay is shown in Fig. 1.

\section{Serum and synovial MMP-3 measurement}

Serum MMP-3 was measured using a latex turbidimetric immunoassay (Eiken Chemical, Tokyo, Japan). Synovial fluid MMP-3 was measured by enzyme-linked immunosorbent assay (R\&D Systems) according to the manufacturer's instructions.

\section{Statistics}

Correlation analysis was conducted using the Pearson product-moment correlation coefficient (JMP 11 software; SAS Institute, Cary, NC, USA). We considered $p<0.05$ to be significant.

\section{Results}

\section{MMP-3 has an 0-glycan cluster}

MMP-3 is a serum protein present in relatively low abundance $(10-100 \mathrm{ng} / \mathrm{ml}$ in healthy people and $50-1000 \mathrm{ng} / \mathrm{ml}$ in patients with RA) [11]. Therefore, MMP-3 was enriched from precleared serum $(40 \mu \mathrm{l})$ of 24 patients with RA by IP (Table 1). To reduce contaminant, we carefully selected streptavidin beads [22] and used anti-MMP-3 antibody after protein $G$ purification, and removed the antibody from IP eluate. Although small amounts of contaminated protein remained in IP samples, we confirmed that the contaminant did not inhibit lectin signals by spike testing using recombinant MMP-3. The yield and stability of IP samples was checked by Western blot analysis (Fig. 2a), and we confirmed that there were no extra bands in all samples. Because we used the same antibody for Western blot analysis and antibody-overlay lectin microarray experiments, almost all lectin signals were thought to be derived from MMP-3. Aliquots of the IP samples, including approximately $1 \mathrm{ng}$ of MMP-3, were subjected to antibody-overlay lectin microarray.

Representative results from two patients, one with high disease activity and the other in remission, are shown in Fig. 2b. Although trace amounts of MMP-3 were applied, four lectins showed significant signal in all samples: Agrocybe cylindracea galectin (ACG), Agaricus bisporus agglutinin (ABA), Amaranthus caudatus agglutinin (ACA),

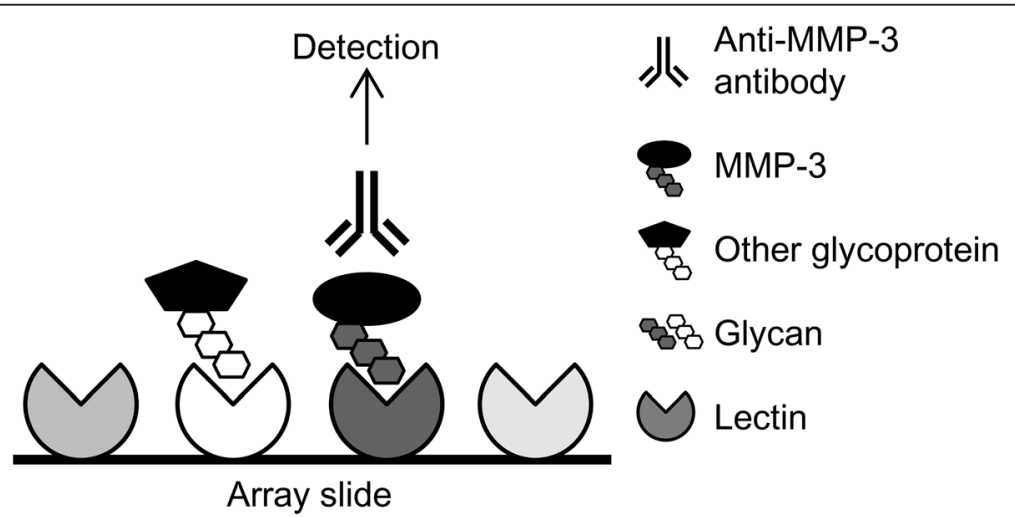

Fig. 1 Schematic overview of the antibody-overlay lectin microarray. A total of 45 kinds of lectins are spotted in triplicate on the array slide. Immunoprecipitation samples were applied to them, and glycoproteins bound to the lectins through their glycans. After washing, the lectins that bound to matrix metalloproteinase (MMP)-3 glycans were detected using anti-MMP-3-specific antibody 
Table 1 Characteristics of the first patient cohort

\begin{tabular}{ll}
\hline Characteristic & First cohort $(n=24)$ \\
\hline Age, years & $68(55-72)$ \\
Female, $n(\%)$ & $20(83)$ \\
Disease duration, months & $36(6-153)$ \\
Stage I/IIIII/IV, $n$ & $9 / 8 / 2 / 5$ \\
Class 1/2/3/4, $n$ & $5 / 14 / 4 / 0$ \\
RF-positive, $n(\%)$ & $19(79)$ \\
ACPA-positive, $n(\%)$ & $20(87)^{\mathrm{a}}$ \\
Methotrexate use, $n(\%)$ & $13(54)$ \\
Biologic use, $n(\%)$ & $8(33)$ \\
DAS28-ESR & $4.6(3.6-5.8)^{\mathrm{a}}$ \\
\hline
\end{tabular}

$R F$ rheumatoid factor, $A C P A$ anticitrullinated protein antibodies, DAS28-ESR Disease Activity Score in 28 joints with erythrocyte sedimentation rate

Values are median (interquartile range) unless otherwise noted ${ }^{a} n=23$ and Jacalin. ACG recognizes $\alpha-2,3$ sialic acid [23], ABA, $\mathrm{ACA}$, and Jacalin commonly recognize the $\mathrm{T}$ antigen (core 1 structure) of $O$-glycan [21]. Such a unique binding pattern at a low concentration has been observed for mucin-like $O$-glycosylated proteins such as mucin 1 [24], podoplanin [21], and immunoglobulin A (IgA) [20]. The estimated glycan structure of the MMP-3 molecule was discussed later in this article. We did not observe a signal for $\mathrm{N}$-glycan binding, although MMP-3 has a potential $\mathrm{N}$ glycosylation site. It is well known that interaction of a glycoprotein that has a single $\mathrm{N}$-glycan, such as AFP, with lectins is weak and difficult to detect at low analyte concentrations. To our knowledge, this is the first report to describe MMP-3 as having an $\mathrm{O}$-glycan cluster.

\section{ACG/Jacalin ratio reflects RA activity}

We observed differences between lectin signals among patients with RA, prompting us to next evaluate the

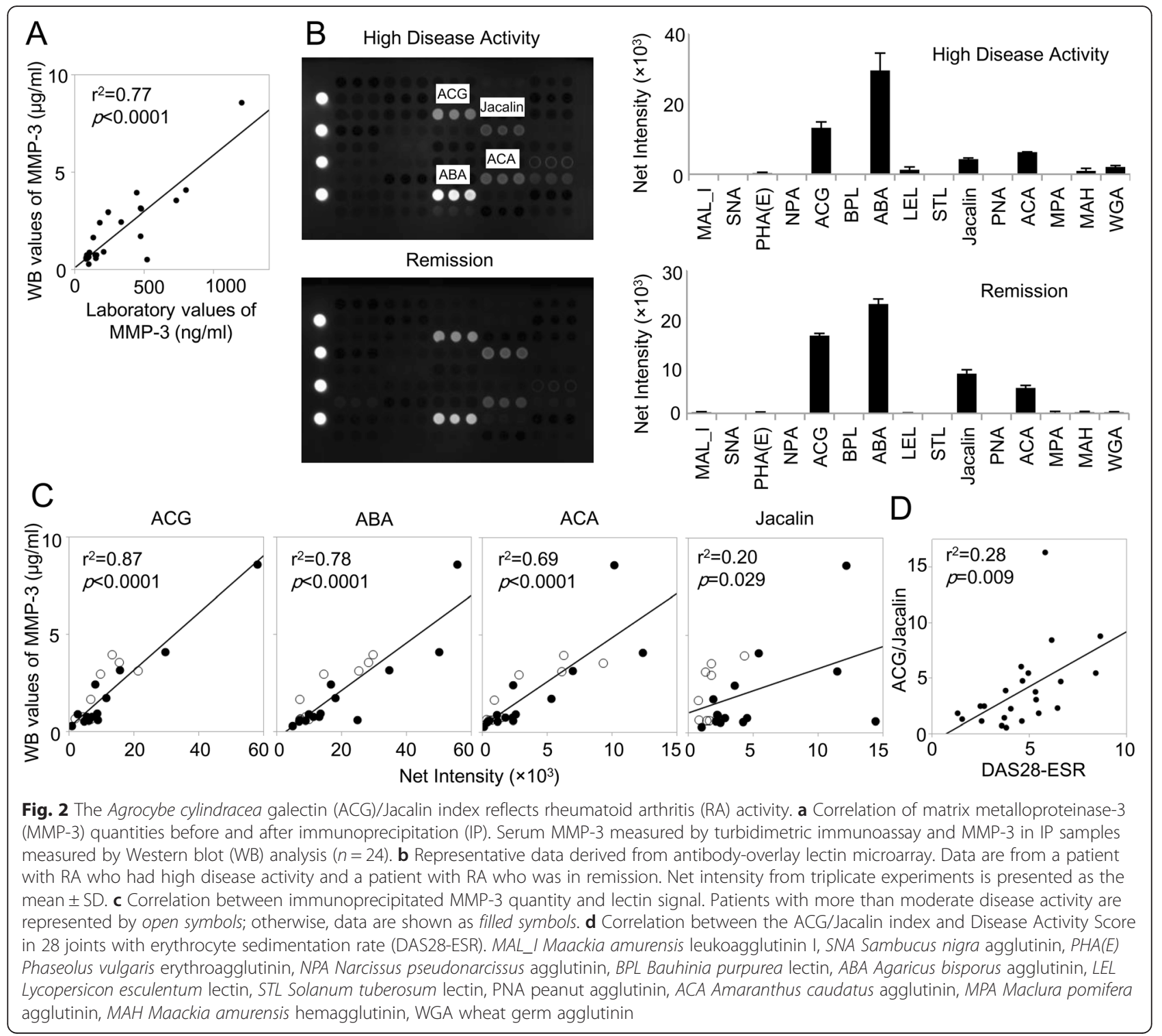


association between lectin signal and RA disease activity. Serum MMP-3 levels varied among patients, with the quantity of MMP-3 that was enriched and analyzed, in addition to the strength of individual lectin signals related to serum MMP-3 concentration, differing. Therefore, we evaluated the correlation between representative lectin signals and MMP-3 quantity (Fig. 2c). The results show that ACG, ABA, and ACA signals correlated well with MMP-3, regardless of RA disease activity. This suggests little variation in the structure of ACG, ABA, and ACA binding glycans. Jacalin signal, however, correlated poorly with MMP-3, suggesting that there were variations in the structure of Jacalin-binding glycans. Especially among patients with a high RA disease activity, Jacalin signals seemed to be relatively low compared with quantity of MMP-3; therefore, we thought it might be possible to use Jacalin as the RA activity biomarker.

However, there are two problems with using Jacalin signals as a disease activity marker: (1) lectin signals is influenced by serum MMP-3 concentration; and (2) because the relationship between disease activity and Jacalin signals was negatively correlated, use of Jacalin signals is impractical. To overcome these problems, we took the ratio of the Jacalin and ACG signals, which had the strongest correlation to MMP-3 quantity. As expected, the ACG/ Jacalin index was positively correlated with RA disease activity (Fig. 2d). This result suggests that profiling MMP-3 glycosylation patterns produced in local RA lesions may serve as a disease activity marker.

\section{ACG/Jacalin index as an RA disease activity marker}

To confirm the association between the ACG/Jacalin index and disease activity, we recruited a second patient cohort and measured the ACG/Jacalin index in the same manner. This validation cohort included 60 serum samples from 30 patients with RA before and after treatment. The median treatment period was 12 months (interquartile range 11-12 months) (Table 2).

The correlation between composite measures and their components and ACG/Jacalin, MMP-3, and CRP are shown in Table 3 . Consistent with previous reports [12, 13], CRP correlated better with DAIs than serum MMP-3. However, focusing on the glycosylation pattern, the ACG/ Jacalin index correlated well with disease activity, similar to CRP. This result indicates that the ACG/Jacalin index may serve as a disease activity marker.

Importantly, the ACG/Jacalin index correlated with each component of the composite measures. In particular, the correlation coefficient was high for the joint score and Physician Global Assessment. The correlations with CRP and MMP-3 were not strong, suggesting they may reflect different aspects of disease activity. Because the standard serum MMP-3 value in males and females is different, MMP-3 values were calculated using only
Table $\mathbf{2}$ Characteristics of the second patient cohort

\begin{tabular}{|c|c|c|}
\hline \multirow[t]{2}{*}{ Characteristic } & \multicolumn{2}{|c|}{ Second patient cohort $(n=60)$} \\
\hline & Pretreatment $(n=30)$ & Posttreatment $(n=30)$ \\
\hline Age, years & $56(37-64)$ & \\
\hline Female, $n(\%)$ & $26(88)$ & \\
\hline Disease duration, months & $13(3-47)$ & \\
\hline Stage $|/| I / I I / I V, n$ & $14 / 14 / 0 / 2$ & \\
\hline Class 1/2/3/4, $n$ & $9 / 19 / 2 / 0$ & \\
\hline RF-positive, $n(\%)$ & $22(73)$ & \\
\hline ACPA-positive, $n$ (\%) & $22(73)$ & \\
\hline Methotrexate use, $n(\%)$ & $18(60)$ & $28(93)$ \\
\hline Biologic use, $n$ (\%) & $5(17)$ & $21(70)$ \\
\hline DAS28-ESR & $4.8(3.7-6.4)$ & $2.0(1.0-2.8)$ \\
\hline DAS28-CRP & $4.0(3.2-5.5)$ & $1.5(1.1-2.3)$ \\
\hline SDAl & $16.0(11.5-32.9)$ & $1.8(0.3-5.4)$ \\
\hline CDAl & $15.4(10.8-31.2)$ & $1.8(0.3-5.4)$ \\
\hline SJC, 28 joints, $n$ & $4(3-8.5)$ & $0(0-1)$ \\
\hline TJC, 28 joints, $n$ & $6(3-10)$ & $0(0-1)$ \\
\hline PtGA, mm & $48(27-73)$ & $35(11-53)$ \\
\hline PGA, mm & $31(20-52)$ & $2(0-11)$ \\
\hline CRP, mg/dl & $0.5(0.1-1.2)$ & $0.0(0.0-0.1)$ \\
\hline $\mathrm{ESR}, \mathrm{mm} / \mathrm{h}$ & $47(28-67)$ & $8(3-24)$ \\
\hline MMP-3, ng/ml & $79(49-107)$ & $39(24-53)$ \\
\hline HAQ-DI & $0.9(0.3-1.4)$ & $0.1(0-0.4)$ \\
\hline
\end{tabular}

ACPA anticitrullinated protein antibodies, CDAI Clinical Disease Activity Index, CRP C-reactive protein, DAS28 Disease Activity Score in 28 joints, ESR erythrocyte sedimentation rate, $M M P-3$ matrix metalloproteinase-3, $R F$ rheumatoid factor, SDAI Simplified Disease Activity Index, SJC swollen joint count, TJC tender joint count, PtGA patient global assessment, PGA Physician Global Assessment, $H A Q-D I$ Health Assessment Questionnaire Disability Index

Values are median (interquartile range) unless otherwise noted

female data (Table 3). A gender gap was not observed in the relationship between the ACG/Jacalin index and disease activity (Fig. 3).

To examine whether the ACG/Jacalin index reflects a change in disease activity, we assessed the correlation between the change in the ACG/Jacalin index and DAIs before and after treatment (Table 4). Surprisingly, the ACG/Jacalin index reflected the degree of disease activity change better than MMP-3 and CRP. Similar to previous results, a change in the ACG/Jacalin index was correlated with a change in each component of the composite measures. These results strongly suggest that the ACG/Jacalin index may serve as a useful RA disease activity marker.

\section{Local RA lesions produce MMP-3 with elevated ACG/Jacalin index}

We found that serum MMP-3 glycosylation patterns reflected RA disease activity, and we further examined synovial fluid MMP-3 to confirm that this change was 
Table 3 Correlation between disease activity markers, disease activity indices, and their components

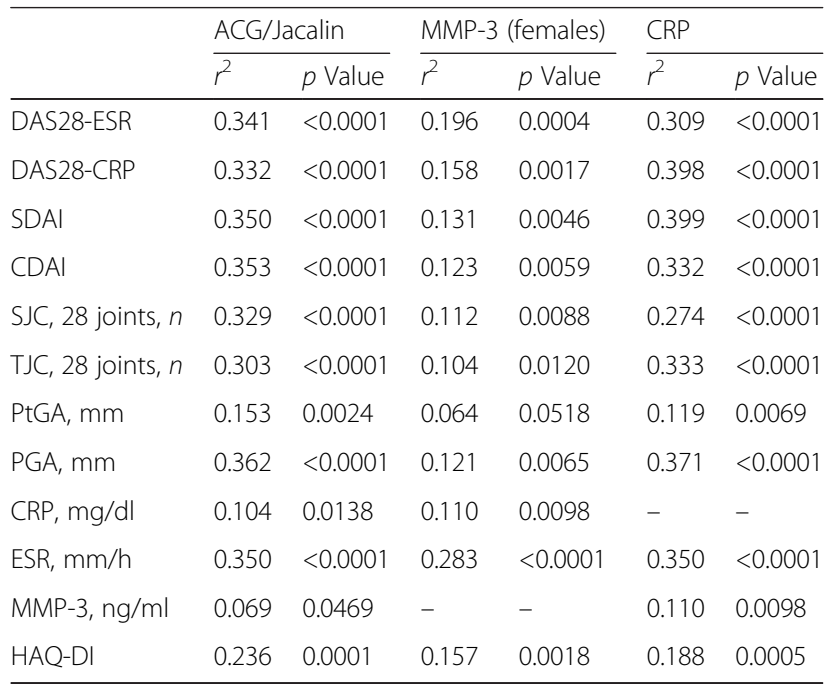

ACG Agrocybe cylindracea galectin, CDAI Clinical Disease Activity Index, CRP C-reactive protein, DAS28 Disease Activity Score in 28 joints, ESR erythrocyte sedimentation rate, HAQ-DI Health Assessment Questionnaire Disability Index, MMP-3 matrix metalloproteinase-3, PGA Physician Global Assessment, PtGA patient global assessment, SDAI Simplified Disease Activity Index, SJC swollen joint count, TJC tender joint count

Pearson product-moment correlation values are shown

derived from local RA lesions. Synovial MMP-3 was immunoprecipitated from untreated patients with active $\mathrm{RA}$ and control patients with OA.

Patient background data and synovial fluid MMP-3 concentrations are shown in Table 5. Synovial MMP-3 was 100 -fold higher than serum MMP-3 and higher in RA versus OA, consistent with previous reports $[9,11]$. To focus on glycosylation differences, an IP and lectin microarray assay was performed after making MMP-3 concentrations equal by dilution. Synovial IP sample yields were confirmed by Western blot analysis (Fig. 4a), with representative lectin signals from three patients with RA and three patients with OA (Fig. 4b). RA synovial fluid MMP-3 had a high ACG signal and a low Jacalin signal compared with synovial fluid of patients with OA, the same as for high RA disease activity as indicated by serum MMP-3. This result suggests that the increase in the ACG/Jacalin index resulted in part from MMP-3 produced in inflammatory RA lesions.

\section{ACG/Jacalin reflects the degree of sialylation}

Jacalin recognizes the $\mathrm{T}$ antigen but does not bind the $\alpha$-2,6-modification of the core $N$-acetylgalactosamine (GalNAc) structure (Fig. 4c) [21]. Therefore, we hypothesized that the difference in ACG/Jacalin signal might indicate the degree of $O$-glycan $\alpha-2,6$-sialylation. To confirm this, we analyzed synovial IP samples by antibody-overlay lectin microarray after sialic acid digestion. As expected, this treatment increased Jacalin and decreased ACG signal, with the Jacalin signal being equivalent after digestion (Fig. 4d). ACG recognizes the Sia- $\alpha-2,3-$ Gal- $\beta-1,3$ GalNAc structure [23]. Thus, these results indicate that MMP-3 $O$-glycan may have a basic structure (Fig. 4e), with the degree of $\alpha-2,6$-sialylation on the core GalNAc structure reflected by the ACG/Jacalin ratio.

Taken together, MMP-3 O-glycan is highly $\alpha-2,6$ sialylated in the RA joint lesion, and the ACG/Jacalin index, which reflects changes in sialylation, may serve as a new RA disease activity biomarker.

\section{Discussion}

Abnormal glycosylation has been studied extensively in RA. Studies have shown that RA exhibits an increase in agalactosyl IgG [25]; a decrease in the GalNAc content
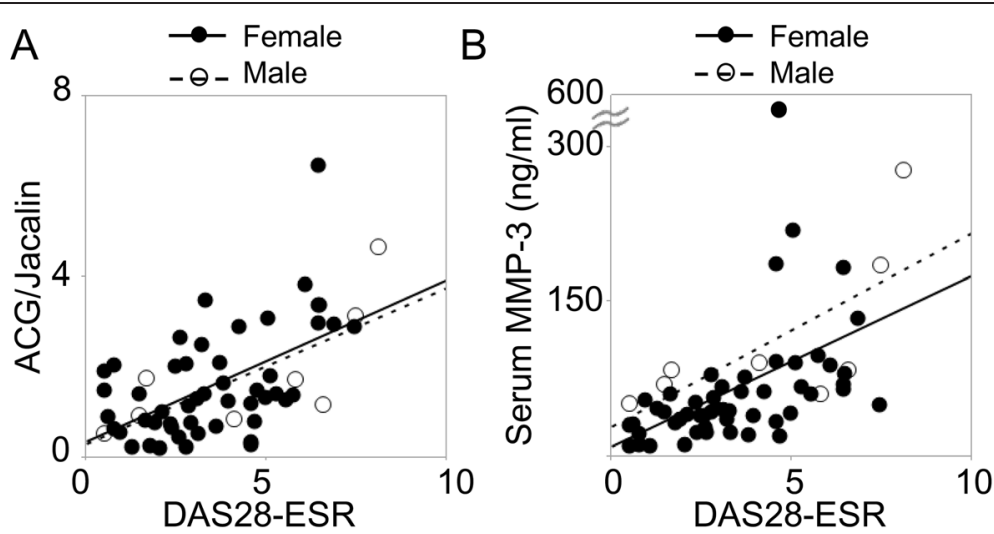

Fig. 3 The Agrocybe cylindracea galectin (ACG)/Jacalin index is similar between males and females. Correlations between disease activity indices and (a) ACG/Jacalin and (b) serum matrix metalloproteinase-3 (MMP-3). Data from female and male patients are shown in filled and open symbols, respectively. DAS28-ESR Disease Activity Score in 28 joints with erythrocyte sedimentation rate 
Table 4 Correlation between the change of disease activity markers, disease activity indices, and their components

\begin{tabular}{|c|c|c|c|c|c|c|}
\hline & \multicolumn{2}{|c|}{$\triangle \mathrm{ACG} /$ Jacalin } & \multicolumn{2}{|c|}{$\Delta \mathrm{MMP}-3$ (females) } & \multicolumn{2}{|l|}{$\triangle C R P$} \\
\hline & $\overline{r^{2}}$ & $\overline{p \text { Value }}$ & $\overline{r^{2}}$ & $\overline{p \text { Value }}$ & $\overline{r^{2}}$ & $p$ Value \\
\hline$\triangle \mathrm{DAS} 28-\mathrm{ESR}$ & 0.321 & 0.0017 & 0.028 & 0.4114 & 0.124 & 0.0564 \\
\hline$\triangle \mathrm{DAS} 28-\mathrm{CRP}$ & 0.254 & 0.0062 & 0.005 & 0.7334 & 0.159 & 0.0292 \\
\hline$\triangle \mathrm{SDAl}$ & 0.245 & 0.0074 & $<0.001$ & 0.9379 & 0.178 & 0.0203 \\
\hline$\triangle \mathrm{CDAl}$ & 0.241 & 0.0080 & $<0.001$ & 0.8952 & 0.116 & 0.0651 \\
\hline$\Delta \mathrm{SJC}, 28 \mathrm{jc}$ & 0.174 & 0.0271 & $<0.001$ & 00215 & 0.152 & 0.0330 \\
\hline$\Delta \mathrm{TJC}, 28$ joints, $n$ & 0.180 & 0.0243 & 0.003 & 0.8083 & 0.109 & 0.0754 \\
\hline$\Delta \mathrm{PtGA}, \mathrm{mm}$ & 0.172 & 0.0280 & 0.002 & 0.8092 & 0.001 & 0.8884 \\
\hline$\triangle \mathrm{PGA}, \mathrm{mm}$ & 0.215 & 0.0130 & 0.006 & 0.7076 & 0.170 & 0.0236 \\
\hline$\Delta \mathrm{CRP}, \mathrm{mg} / \mathrm{dl}$ & 0.055 & 0.2293 & 0.023 & 0.4553 & - & - \\
\hline$\Delta \mathrm{ESR}, \mathrm{mm} / \mathrm{h}$ & 0.468 & $<0.0001$ & 0.200 & 0.0218 & 0.217 & 0.0094 \\
\hline$\Delta \mathrm{MMP}-3, \mathrm{ng} / \mathrm{ml}$ & 0.093 & 0.1143 & - & - & 0.010 & 0.6074 \\
\hline$\Delta \mathrm{HAQ}-\mathrm{DI}$ & 0.217 & 0.0125 & 0.012 & 0.5896 & 0.001 & 0.9000 \\
\hline
\end{tabular}

ACG Agrocybe cylindracea galectin, CDAl Clinical Disease Activity Index, CRP C-reactive protein, DAS28 Disease Activity Score in 28 joints, ESR erythrocyte sedimentation rate, HAQ-DI Health Assessment Questionnaire Disability Index, MMP-3 matrix metalloproteinase-3, PGA Physician Global Assessment, PtGA patient global assessment, SDAI Simplified Disease Activity Index, SJC swollen joint count, TJC tender joint count

Pearson product-moment correlation values are shown

of IgA [26]; and more recently, extensive glycosylation of the anticitrullinated protein antibodies-IgG variable domain [27]. Notably, most were surrogate markers that were not expressed in diseased synovial tissue. Our data point to a new RA activity marker using a change in glycosylation of a glycoprotein directly expressed in, and secreted from, synovial tissue.

Although CRP and MMP-3 are widely used to monitor RA, they do not demonstrate sufficient specificity to determine disease activity. Thus, RA disease activity markers, such as the multibiomarker disease activity test $[28,29]$ and the leucine-rich $\alpha 2$ glycoprotein [30], were developed. However, it has been difficult to find a protein that specifically reflects RA disease activity. In this study, we focused on the glycan content of an existing marker, MMP-3, to determine both specificity and sensitivity metrics.

As previously mentioned, glycosylation is influenced by the state of the cell [14]. MMP-3 production is increased in joint synovitis and is continually produced even in noninflamed joints. However, our results show that MMP-3 glycans change under inflammatory conditions and that the ACG/Jacalin index seemed to distinguish disease-specific MMP-3 from total MMP-3. This is one reason why our marker correlated well with disease activity. In addition, MMP-3 expression is relatively restricted within the joint and partly leaks into the serum [11]. Thus, it might be possible to directly determine inflammation at local lesions without an effect of any other influences. Interestingly, the ACG/Jacalin index had a higher correlation coefficient for joint parameters than CRP or MMP-3, suggesting that this index is a more specific arthritis marker.

The ACG/Jacalin index has advantages as a disease activity marker, but further study is required. The method used to obtain lectin signals is slightly complicated, and thus a simplified method is needed. It is better to clarify the glycan structure of MMP-3 and to detect MMP-3 with altered glycan directly. Additionally, sensitivity and stability should be improved for practical use because serum MMP-3 concentration is quite low in some patients after treatment. Although serum MMP-3 was reported to be a radiological predictor [31-33], we could not examine the association between ACG/Jacalin index and radiological progression, owing to the limited number of patients. In addition to RA, MMP-3 glycans may be altered in other disease conditions that increase serum MMP-3, such as psoriatic arthritis, reactive arthritis, steroid use, and polymyalgia rheumatica [10, 31], requiring measurement of $\mathrm{ACG} / J a c a l i n$ under various situations.

To our knowledge, this study shows for the first time that serum glycoproteins exhibiting disease-associated glycosylation changes have potential as disease-specific biomarkers in autoimmune diseases. In patients with RA, differences in serum MMP-3 $\alpha$-2,6-sialylation may be a

Table 5 Characteristics of patients who provided synovial fluid samples

\begin{tabular}{lllllr}
\hline Patient & Disease & Age (years) & Sex & DAS28-ESR & Synovial fluid MMP-3 $(\mu \mathrm{g} / \mathrm{ml})$ \\
\hline 1 & RA & 71 & Male & 5.12 & 45.5 \\
2 & RA & 87 & Female & 6.90 & 11.0 \\
3 & RA & 77 & Female & 4.88 & 32.0 \\
4 & OA & 79 & Female & - & 0.7 \\
5 & OA & 81 & Female & - & 1.3 \\
6 & OA & 67 & Female & - & 2.4 \\
\hline
\end{tabular}

DAS28 Disease Activity Score in 28 joints, ESR erythrocyte sedimentation rate, MMP-3 matrix metalloproteinase-3, OA osteoarthritis, RA rheumatoid arthritis 


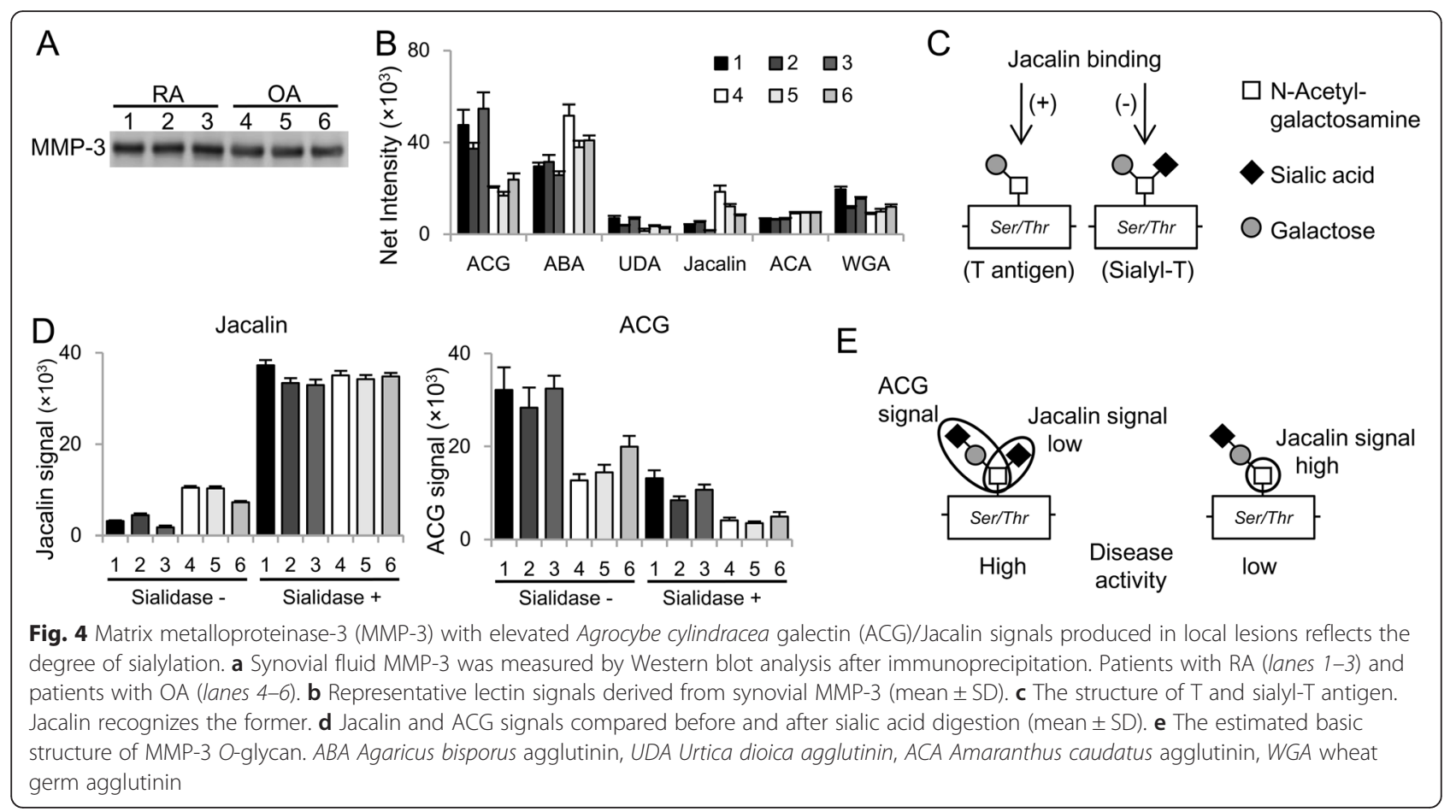

useful index for estimating disease activity. Further studies are needed to validate this index and to develop new glycoprotein biomarkers in other autoimmune diseases.

\section{Conclusions}

We have developed a new, sensitive RA-specific biomarker based on MMP-3 glycosylation changes. The ratio of two lectin signals, ACG and Jacalin, correlated well with several DAIs and their components and also reflected DAI changes following RA treatment. Further studies are required to develop the practical use of this biomarker.

\section{Ethical approval and consent to participate}

This study was approved by the institutional review board of Keio University School of Medicine and the National Institute of Advanced Industrial Science and Technology, and it was conducted in compliance with the Declaration of Helsinki. Written informed consent was obtained from the patients for publication of their individual details and accompanying images in this article. The consent forms are held by the authors and are available for review by the Editor-in-Chief of this journal.

\section{Abbreviations}

ABA: Agaricus bisporus agglutinin; ACA: Amaranthus caudatus agglutinin; ACG: Agrocybe cylindracea galectin; ACPA: anticitrullinated protein antibodies; BPL: Bauhinia purpurea lectin; AFP: a-fetoprotein; CDAl: Clinical Disease Activity Index; CRP: C-reactive protein; DAl: disease activity index; DAS28: Disease Activity Score in 28 joints; ESR: erythrocyte sedimentation rate; GalNAc: N-acetylgalactosamine; HAQ-DI: Health Assessment
Questionnaire Disability Index; IgA: immunoglobulin A; IgG: immunoglobulin G; IP: immunoprecipitation; IQR: interquartile range; LEL: Lycopersicon esculentum lectin; MAH: Maackia amurensis hemagglutinin; MAL_I: Maackia amurensis leukoagg lutinin I; MMP-3: matrix metalloproteinase-3; MPA: Maclura pomifera agglutinin; NPA: Narcissus pseudonarcissus agglutinin; OA: osteoarthritis; PBS-TX: PBS containing 1 \% Triton X-100; PGA: Physician Global Assessment; PHA(E): Phaseolus vulgaris erythroagglutinin; PNA: peanut agglutinin;

PtGA: patient global assessment; RA: rheumatoid arthritis; RF: rheumatoid factor; SDAl: Simplified Disease Activity Index; SJC: swollen joint count; SNA: Sambucus nigra agglutinin; STL: Solanum tuberosum lectin; TBS-TX: Tris-buffered saline containing $1 \%$ Triton X-100; TJC: tender joint count; UDA: Urtica dioica agglutinin; WGA: wheat germ agglutinin.

\section{Competing interests}

The authors declare that they have no competing interests.

\section{Authors' contributions}

MT and AK participated in the design of the study, collected and analyzed data, and drafted and revised the manuscript. KS participated in the design of the study and helped to draft and revise the manuscript. AM, YK, MS, and HN participated in the design of the study and revised the manuscript. HS, $\mathrm{TN}$, and YO collected and analyzed data, and helped to draft the manuscript. $\Pi T$ participated in the design and coordination of the study and revised the manuscript. All authors read and approved the manuscript.

\section{Acknowledgements}

We especially thank Harumi Kondo and Mayumi Ota for helping with the acquisition of clinical information.

\section{Funding}

This work was supported by an institutional research grant from Keio University, the Center of Innovation Trial Program of the Japan Science and Technology Agency, and the Tailor-made Medical Treatment Program (BioBank Japan Project) of the Ministry of Education, Culture, Sports, Science, and Technology of Japan.

\section{Author details}

'Division of Rheumatology, Department of Internal Medicine, Keio University School of Medicine, 35 Shinanomachi, Shinjuku-ku, Tokyo 160-8582, Japan. 
${ }^{2}$ Research Center for Medical Glycoscience (RCMG), National Institute of Advanced Industrial Science and Technology (AIST), 1-1-1 Umezono, Tsukuba, Ibaraki 305-8568, Japan. ${ }^{3}$ Center for Systems Medicine, Keio University School of Medicine, 35 Shinanomachi, Shinjuku-ku, Tokyo 160-8582, Japan. ${ }^{4}$ Department of Biochemistry, Keio University School of Medicine, 35 Shinanomachi, Shinjuku-ku, Tokyo 160-8582, Japan.

Received: 24 February 2016 Accepted: 4 May 2016

Published online: 21 May 2016

\section{References}

1. Mclnnes IB, Schett $G$. The pathogenesis of rheumatoid arthritis. N Engl J Med. 2011;365:2205-19.

2. Schoels M, Knevel R, Aletaha D, Bijlsma JW, Breedveld FC, Boumpas DT, et al. Evidence for treating rheumatoid arthritis to target: results of a systematic literature search. Ann Rheum Dis. 2010;69:638-43.

3. Smolen JS, Breedveld FC, Burmester GR, Bykerk V, Dougados M, Emery P, et al. Treating rheumatoid arthritis to target: 2014 update of the recommendations of an international task force. Ann Rheum Dis. 2016;75:3-15.

4. van Gestel AM, Haagsma CJ, van Riel PL. Validation of rheumatoid arthritis improvement criteria that include simplified joint counts. Arthritis Rheum. 1998;41:1845-50.

5. Prevoo ML, van 't Hof MA, Kuper $H H$, van Leeuwen $M A$, van de Putte $L B$, van Riel PL. Modified disease activity scores that include twenty-eight-joint counts: development and validation in a prospective longitudinal study of patients with rheumatoid arthritis. Arthritis Rheum. 1995;38:44-8.

6. Smolen JS. A simplified disease activity index for rheumatoid arthritis for use in clinical practice. Rheumatology. 2003;42:244-57.

7. Aletaha D, Nell VP, Stamm T, Uffmann M, Pflugbeil S, Machold K, et al. Acute phase reactants add little to composite disease activity indices for rheumatoid arthritis: validation of a clinical activity score. Arthritis Res Ther. 2005;7:R796-806.

8. Okada $Y$, Nagase $H$, Harris Jr ED. A metalloproteinase from human rheumatoid synovial fibroblasts that digests connective tissue matrix components: purification and characterization. J Biol Chem. 1986;261:14245-55.

9. Yoshihara Y, Nakamura H, Obata K, Yamada H, Hayakawa T, Fujikawa K, et al. Matrix metalloproteinases and tissue inhibitors of metalloproteinases in synovial fluids from patients with rheumatoid arthritis or osteoarthritis. Ann Rheum Dis. 2000;59:455-61.

10. Ribbens C, Martin y Porras M, Franchimont N, Kaiser MJ, Jaspar JM, Damas P, et al. Increased matrix metalloproteinase-3 serum levels in rheumatic diseases: relationship with synovitis and steroid treatment. Ann Rheum Dis. 2002;61:161-6.

11. Taylor DJ, Cheung NT, Dawes PT. Increased serum proMMP-3 in inflammatory arthritides: a potential indicator of synovial inflammatory monokine activity. Ann Rheum Dis. 1994:53:768-72.

12. Keyszer G, Lambiri I, Nagel R, Keysser C, Keysser M, Gromnica-Ihle E, et al. Circulating levels of matrix metalloproteinases MMP-3 and MMP-1, tissue inhibitor of metalloproteinases 1 (TIMP-1), and MMP-1/TIMP-1 complex in rheumatic disease. Correlation with clinical activity of rheumatoid arthritis versus other surrogate markers. J Rheumatol. 1999;26:251-8.

13. Ateş A, Türkçapar N, Olmez U, Tiryaki O, Düzgün N, Uğuz E, et al. Serum pro-matrix metalloproteinase-3 as an indicator of disease activity and severity in rheumatoid arthritis: comparison with traditional markers. Rheumatol Int. 2007;27:715-22

14. Kuno A, Matsuda A, Unno S, Tan B, Hirabayashi J, Narimatsu H. Differential glycan analysis of an endogenous glycoprotein: toward clinical implementation — from sample pretreatment to data standardization. Methods Mol Biol. 2014;1200:265-85.

15. Hayashi K, Kumada T, Nakano S, Takeda I, Sugiyama K, Kiriyama S, et al. Usefulness of measurement of Lens culinaris agglutinin-reactive fraction of a-fetoprotein as a marker of prognosis and recurrence of small hepatocellular carcinoma. Am J Gastroenterol. 1999;94:3028-33.

16. Badr HA, Alsadek DM, Darwish AA, Elsayed Al, Bekmanov BO, Khussainova EM, et al. Lectin approaches for glycoproteomics in FDA-approved cancer biomarkers. Expert Rev Proteomics. 2014;11:227-36.

17. Kuno A, Ikehara Y, Tanaka Y, Ito K, Matsuda A, Sekiya S, et al. A serum "sweet-doughnut" protein facilitates fibrosis evaluation and therapy assessment in patients with viral hepatitis. Sci Rep. 2013;3:1065.
18. Grassadonia A, Tinari N, lurisci I, Piccolo E, Cumashi A, Innominato P, et al. $90 \mathrm{~K}$ (Mac-2 BP) and galectins in tumor progression and metastasis. Glycoconj J. 2004;19:551-6.

19. Aletaha D, Neogi T, Silman AJ, Funovits J, Felson DT, Bingham 3rd CO, et al. 2010 rheumatoid arthritis classification criteria: an American College of Rheumatology/European League Against Rheumatism collaborative initiative. Ann Rheum Dis. 2010;69:1580-8.

20. Narimatsu Y, Kuno A, Ito H, Kaji H, Kaneko S, Usui J, et al. IgA nephropathy caused by unusual polymerization of IgA1 with aberrant $N$-glycosylation in a patient with monoclonal immunoglobulin deposition disease. PLoS One. 2014;9:e91079.

21. Kuno A, Kato Y, Matsuda A, Kaneko MK, Ito H, Amano K, et al. Focused differential glycan analysis with the platform antibody-assisted lectin profiling for glycan-related biomarker verification. Mol Cell Proteomics. 2009;8:99-108.

22. Nishio K, Masaike Y, Ikeda M, Narimatsu H, Gokon N, Tsubouchi S, et al. Development of novel magnetic nano-carriers for high-performance affinity purification. Colloids Surf B Biointerfaces. 2008;64:162-9.

23. Yagi F, Miyamoto M, Abe T, Minami Y, Tadera K, Goldstein IJ. Purification and carbohydrate-binding specificity of Agrocybe cylindracea lectin. Glycoconj J. 1997;14:281-8.

24. Matsuda A, Kuno A, Nakagawa T, Ikehara Y, Irimura T, Yamamoto M, et al. Lectin microarray-based sero-biomarker verification targeting aberrant O-linked glycosylation on mucin 1. Anal Chem. 2015;87:7274-81.

25. Parekh RB, Dwek RA, Sutton BJ, Fernandes DL, Leung A, Stanworth D, et al. Association of rheumatoid arthritis and primary osteoarthritis with changes in the glycosylation pattern of total serum IgG. Nature. 1985;316:452-7.

26. Wada Y, Tajiri M, Ohshima S. Quantitation of saccharide compositions of O-glycans by mass spectrometry of glycopeptides and its application to rheumatoid arthritis. J Proteome Res. 2010;9:1367-73.

27. Rombouts $Y$, Willemze A, van Beers JJ, Shi J, Kerkman PF, van Toorn L, et al. Extensive glycosylation of ACPA-lgG variable domains modulates binding to citrullinated antigens in rheumatoid arthritis. Ann Rheum Dis. 2016;75:578-85.

28. Curtis JR, van der Helm-van Mil AH, Knevel R, Huizinga TW, Haney DJ, Shen Y, et al. Validation of a novel multibiomarker test to assess rheumatoid arthritis disease activity. Arthritis Care Res (Hoboken). 2012;64:1794-803.

29. Centola M, Cavet G, Shen Y, Ramanujan S, Knowlton N, Swan KA, et al. Development of a multi-biomarker disease activity test for rheumatoid arthritis. PLoS One. 2013;8, e60635.

30. Serada S, Fujimoto M, Ogata A, Terabe F, Hirano T, lijima H, et al. iTRAQbased proteomic identification of leucine-rich a-2 glycoprotein as a novel inflammatory biomarker in autoimmune diseases. Ann Rheum Dis. 2010;69:770-4.

31. Posthumus MD, Limburg PC, Westra J, Cats HA, Stewart RE, van Leeuwen MA, et al. Serum levels of matrix metalloproteinase-3 in relation to the development of radiological damage in patients with early rheumatoid arthritis. Rheumatology (Oxford). 1999;38:1081-7.

32. Young-Min S, Cawston T, Marshall N, Coady D, Christgau S, Saxne T, et al. Biomarkers predict radiographic progression in early rheumatoid arthritis and perform well compared with traditional markers. Arthritis Rheum. 2007:56:3236-47.

33. Houseman M, Potter C, Marshall N, Lakey R, Cawston T, Griffiths I, et al. Baseline serum MMP-3 levels in patients with rheumatoid arthritis are still independently predictive of radiographic progression in a longitudinal observational cohort at 8 years follow up. Arthritis Res Ther. 2012;14:R30

\section{Submit your next manuscript to BioMed Central and we will help you at every step:}

- We accept pre-submission inquiries

- Our selector tool helps you to find the most relevant journal

- We provide round the clock customer support

- Convenient online submission

- Thorough peer review

- Inclusion in PubMed and all major indexing services

- Maximum visibility for your research

Submit your manuscript at www.biomedcentral.com/submit 